\title{
Un nouveau chantier d'archives orales : l'histoire des décisions stratégiques de la SNCF et de leur application depuis les années 1970
}

Jean-Pierre Williot

\section{OpenEdition}

Journals

Édition électronique

URL : https://journals.openedition.org/rhcf/685

DOI : 10.4000/rhcf.685

Éditeur

Rails \& histoire

Édition imprimée

Date de publication : 1 mai 2004

Pagination : 29-61

ISSN : 0996-9403

\section{Référence électronique}

Jean-Pierre Williot, « Un nouveau chantier d'archives orales : I'histoire des décisions stratégiques de la SNCF et de leur application depuis les années $1970 »$, Revue d'histoire des chemins de fer [En ligne], 31 2004, mis en ligne le 07 avril 2011, consulté le 22 avril 2022. URL : http://journals.openedition.org/ rhcf/685; DOI : https://doi.org/10.4000/rhcf.685 


\section{Un nouveau chantier d'archives orales : I'histoire des décisions stratégiques de la SNCF et de leur application depuis les années 1970}

\section{Introduction}

Dans l'article d'orientations problématiques qu'il consacra à «Histoire économique et chemin de fer», François Caron écrivait à l'automne 1989 que cette histoire devait comprendre plusieurs approches dont « l'histoire interne des stratégies et de leurs fondements conceptuels $»^{1}$. Nous sommes dans cette perspective avec la mise en œuvre d'un programme d'archives orales consacrées à l'histoire des décisions stratégiques de la SNCF et de leur application depuis les années 1970. Décidée par le Comité scientifique de l'AHICF, cette grande enquête doit permettre d'atteindre trois objectifs, énoncés par ordre croissant d'importance. Elle est en premier lieu une illustration supplémentaire des missions de l'association qui confirme ainsi son rang parmi les institutions patrimoniales ayant engagé des campagnes d'archives orales dans les dernières décennies ${ }^{2}$. Après avoir piloté un programme thématique sur «Les origines des grandes vitesses ferroviaires en France » confié au CNRS en 1990-1992 et avoir réalisé une collecte plus limitée sur la SNCF et les cheminots dans la guerre 1939-1945 en 1999-2000, l'AHICF poursuit la recherche, la préservation et la création de sources d'histoire ferroviaire. L'étude des décisions stratégiques s'intégrera ainsi dans la formation plus large d'un patrimoine sonore spécifique. Par ailleurs, cette quête scientifique d'informations pour mieux comprendre les évolutions de la SNCF depuis trente ans

1 - François Caron, «Histoire économique et chemin de fer », Revue d'bistoire des chemins de fer, $\mathrm{n}^{\circ} 1$ (automne 1989), p. 25.

2 - Nous renvoyons à l'ouvrage de synthèse de Florence Descamps, L'Historien, l'archiviste et le magnétophone. De la constitution de la source orale à son exploitation, Paris, Comité pour l'histoire économique et financière de la France, 2001, 864 p. L'auteur dresse un panorama précis de la constitution progressive d'archives orales comme conséquence de l'essor de l'histoire orale. Voir en particulier p. 151-222. 
est une occasion unique de conserver une mémoire, fatalement fugace, auprès des grands acteurs de cette période. Elle constitue à la fois une lecture individuelle d'un passé récent et une mise en perspective collective d'une culture d'entreprise. Ce programme n'est par conséquent pas figé sur une époque révolue de la SNCF mais sur une approche dynamique de la place de la SNCF dans la France contemporaine. Enfin, par leur mise à la disposition du public selon les normes en vigueur concernant les témoignages enregistrés, ces entretiens ouvrent, complètent ou étayent de nouvelles perspectives de recherche qui dépassent largement l'intérêt que l'on peut porter à la seule SNCF. Le corpus qui sera réuni a vocation à devenir un observatoire des mutations territoriales et sociales de la France des trente dernières années. On ne peut en effet dissocier la période choisie de changements structurels qui ont affecté l'Hexagone depuis 1970 : développement des pouvoirs régionaux, redistribution des pôles industriels, dynamisme des métropoles d'équilibre, demande d'une plus grande mobilité citadine et interurbaine, nouveaux maillages infrastructurels du territoire. Indépendamment, les dates retenues pour encadrer ce programme ont leur logique. Le point de départ fixé au début de la décennie 1970 se justifie a priori par la mise en œuvre d'une réforme des structures à la SNCF entre 1969 et $1972^{3}$. Le terme, fixé au début des années 2000, permet d'intégrer à la fois les effets de la création de Réseau ferré de France et d'examiner un premier bilan de la régionalisation ferroviaire ${ }^{5}$.

Volet d'un programme de collecte d'archives orales qui a été défini en 2002 sur le thème général du "personnel de la SNCF et son entreprise 1937-2002 », l'axe portant sur les décisions stratégiques et les évolutions des structures de l'entreprise demandait une définition préliminaire. Celle-ci fondait notre approche de départ. Les termes de

3- La réforme est engagée par un échange de lettres entre le ministre des Transports et le président du conseil d'administration de la SNCF en juillet 1969. Elle est mise en œuvre par un décret du 7 avril 1971 portant approbation de l'avenant du 27 janvier 1971 à la convention modifiée du 31 août 1937 et un décret du 23 décembre 1971 portant approbation du nouveau cahier des charges aménageant sur le plan de l'exploitation le régime d'autonomie de gestion conféré à la SNCF. Par ailleurs, le $1^{\text {er }}$ janvier 1973, une réforme des structures de la SNCF est appliquée au moyen d'une décentralisation des décisions qui entraîne la suppression des anciens services et arrondissements correspondants, puis la création de 25 régions (aujourd'hui 23).

4- RFF a été créé par la loi du 13 février 1997 (avec effet rétroactif au $1^{\text {er }}$ janvier).

5- Depuis le $1^{\text {er }}$ janvier 2002, après un long processus de régionalisation, les régions sont autorités organisatrices des transports ferroviaires régionaux de voyageurs, à l'exception de l'Île-de-France et de la Corse. 
décisions stratégiques supposaient de prendre en compte les acteurs en mesure d'orienter ou de peser sur les choix de l'entreprise. Les témoins à solliciter devaient donc avoir été impliqués directement dans une orientation stratégique, soit pour l'avoir initiée, soit pour l'avoir décidée, soit pour en avoir assuré la mise en œuvre au sein de l'entreprise. En qualifiant de stratégiques certaines décisions, nous serions conduits à isoler des événements perçus comme des changements importants, peut-être vécus comme des ruptures, portant en tous cas des objectifs à atteindre. Il en résulte qu'il nous a fallu intégrer dès le départ l'analyse des processus de décision comme un paramètre essentiel et tenter de confronter deux cultures, technique et commerciale, qui, par ingénieurs et cadres interposés, s'opposent parfois et se complètent souvent. Sérier les choix importants, ceux qui ont été des facteurs structurants de la SNCF, reconnus comme des étapes fortes de l'histoire ferroviaire et de celle, conjointe, des cheminots, n'allait cependant pas sans arbitraire. Constituer sur ces bases un corpus de témoins pouvait relever d'une approche trop subjective.

La mise en œuvre d'une étude préliminaire, que nous avons conduite de septembre 2003 à janvier 2004 avec l'assistance technique de deux collaborateurs ${ }^{6}$, sur un panel limité de témoins, a tenté de corriger ce biais. Elle dépasse la réflexion préalable qui fonde toute démarche scientifique pour réunir des archives orales. Elle ne correspond que partiellement à la «maturation historique » présentée comme une condition favorable au démarrage d'archives orales dans la mesure où l'expérience ne procède pas d'une demande de mémoire ${ }^{7}$. Elle repose plutôt sur une prise de conscience externe de l'intérêt d'une collecte. On ne peut nier malgré tout que des évolutions récentes de la SNCF aient joué en faveur d'une réceptivité des témoins sollicités. En quelque sorte année zéro de ce programme de recherche, celle-ci a permis d'établir des points de repères fondamentaux. Ils constituent dorénavant le cadre dans lequel doivent s'inscrire ces archives orales, appelées à se déployer sur plusieurs années. Cette étude apparaît aujourd'hui comme l'essence du programme futur en ce qu'elle a permis de balayer des thèmes

6- Renaud Chaplain, doctorant à l'université Lyon II, a réalisé huit enregistrements ; Natacha Lépine, doctorante à l'université Paris X-Nanterre, a procédé à l'enregistrement de quatre entretiens.

7- Nous reprenons l'expression employée par madame Descamps qui avance comme facteurs favorables à l'entreprise d'un programme d'archives orales : la préexistence de l'ancienneté d'une institution, la complexité d'une organisation, l'instabilité annoncée d’une société. F. Descamps, op. cit., p. 255-261. 
problématiques et de leur assigner des priorités. Elle apparait d'autant plus utile qu'il s'agit de constituer des archives orales et non seulement des sources orales ${ }^{8}$. Elle a également contribué à rendre plus efficace la constitution des cercles de témoins appelés à délivrer ultérieurement leur témoignage. L'expérience que nous en avons tirée conduit à rappeler la démarche et les objectifs qui furent les nôtres. Elle ne saurait en rien prétendre guider des approches similaires. On doit plutôt y lire les prolégomènes d'un livre ouvert, dont les chapitres ont été délimités par les propositions des acteurs.

\section{Au milieu du gué : le « test » de la faisabilité}

\section{La difficile délimitation d'un groupe de témoins de référence}

La difficulté initiale qui se posait à l'orée de l'étude de faisabilité était moins de vérifier l'existence de sources écrites pouvant l'accompagner, déjà bien repérées ${ }^{9}$ que de choisir des témoins. Il ne s'agissait pas d'emblée d'établir une longue liste des acteurs des décisions stratégiques depuis les années 1970. D’un commun accord avec le comité de pilotage qui émane du Comité scientifique de l'AHICF, il a été préconisé de retenir une dizaine de grands témoins pour valider des hypothèses de recherche. Valider ne signifie pas que nous souhaitions placer la problématique sous la tutelle de personnalités et contraindre ainsi notre champ de recherche. Il s'agissait plutôt d'explorer des pistes de travail pour tendre à l'exhaustivité de l'enquête. La démarche supposait donc de réunir des acteurs aux regards différenciés mais suffisamment proches des niveaux décisionnaires pour qu'ils en aient une connaissance précise appuyée sur une information personnelle. La «polyphonie » recommandée dans les campagnes d'archives orales trouve ici une limite en resserrant les cercles concernés. En effet, si à des degrés différents les personnels de la SNCF ont dans leur ensemble pu contribuer à appliquer des réformes structurelles, l'analyse de la prise

\footnotetext{
8- Nous renvoyons sur cette distinction à l'article de D. Voldman, "Définitions et usages », in "La bouche de la vérité ? La recherche historique et les sources orales ", Cabiers de l'IHTP, n 21 (novembre 1992), p. 35. L'archive est un document sonore enregistré par un historien (en l'occurrence), en fonction d'un sujet précis et déposé dans une institution. La source est un matériau recueilli par un chercheur pour étayer son propre travail scientifique, sans perspective de pérennité ni de réutilisation.

9- On peut disposer des archives conservées au Centre d'archives historiques de la SNCF, au Mans, notamment les rapports au conseil d'administration et les rapports de gestion ; des fonds d'archives des différents ministères de tutelle, déposés aux Archives Nationales, en particulier celui en charge de l'équipement et des transports.
} 
de décision ne concerne qu'un nombre limité d'intervenants. Ceux-ci ne sont pas pour autant - ni obligatoirement - les cadres au sommet de la hiérarchie managériale. Certains acteurs, situés à des positions de carrefour d'un processus de décision, ont eu à connaitre des enjeux stratégiques, de leurs difficultés concrètes d'application ou, au contraire, ont apporté la réponse opportune à des besoins diffus.

En conséquence, les témoins que nous avons retenus répondaient à trois critères de sélection. Le premier tombe sous le sens : ils devaient avoir occupé une fonction susceptible de les situer sur un plan spécifique de l'histoire des décisions stratégiques (par exemple l'évolution des structures en charge du transport marchandises pour analyser le déclin du fret, la mise en place de la régionalisation pour restituer l'imbrication des processus qui ont abouti à donner un pouvoir décisionnaire aux régions en matière de transport). Le second critère était d'éviter une redondance avec un autre témoin ayant occupé les mêmes fonctions institutionnelles. Aussi écartions-nous deux directeurs régionaux, deux directeurs généraux ou deux présidents pour n'en retenir qu'un seul, du moins dans cette phase préliminaire. Enfin, nous ne souhaitions pas, dans la mesure du possible, superposer des témoins des mêmes périodes afin d'obtenir des panoramas chronologiques allant de l'amont jusqu'aux années les plus récentes, inscrites dans une histoire du temps présent (pour expliquer la stratégie commerciale actuelle par exemple). De surcroit, la liste devait ouvrir la perception de l'entreprise par le choix croisé de différentes directions internes et par un regard externe.

Les noms choisis l'ont été de manière classique après dépouillement des organigrammes de l'entreprise, prise en compte des notices biographiques et consultation de messieurs Bruno Baufine-Ducrocq, Président Délégué de l'AHICF, et André Blanc, son prédécesseur, aujourd'hui administrateur de l'association, tous deux anciens directeurs à la SNCF. Au total, la sélection effectuée à conduit à retenir quatorze « témoins-source », après avoir constaté la difficulté de s'en tenir à dix comme l'avait à l'origine souhaité le Comité scientifique. La croissance du paneln'est pas anecdotique. Elle caractérise la propension du recours inflationniste au témoignage destiné à valider le choix des thèmes dès que l'on souhaite disposer d'une vision panoramique. Des limites s'imposaient cependant. On a donc écarté volontairement de la liste un certain nombre d'acteurs des années 1970-2000 en partant du principe qu'ils seraient tous interviewés lors de la seconde phase du programme. Déjà repérés comme des témoins essentiels, leur absence n’est donc pas à interpréter comme une moindre estime portée à leur témoignage, ni le résultat d'un choix sélectif dicté par des personnes extérieures au 
programme, comme pouvaient le craindre certains de nos interlocuteurs. Un seul témoin pressenti a refusé l'interview, semble-t-il faute de temps disponible à consacrer à ce programme préliminaire. À l'inverse, on peut se féliciter d'avoir reçu le meilleur accueil auprès d'acteurs en fonction qui ont accepté d'apporter leur analyse des problématiques suggérées. Ils ont validé du même coup l'intérêt d'une étude allant jusqu'à la période la plus récente et l'on peut souhaiter que leur témoignage suscite a posteriori une dynamique qui pérennise cette campagne d'archives orales.

Les quatorze témoins qui ont accepté de livrer un premier témoignage ont, par ordre chronologique des entretiens, été interrogés sur leur action dans les fonctions suivantes :

- fonctions à la direction des Transports terrestres du ministère de l'Équipement, des Transports et du Logement (1986-1997) ; secrétaire du conseil d'administration de RFF, 1997-2000 ;

- chef du Service de l'action régionale de la SNCF, 1983-2000;

- directeur commercial marchandises de la SNCF, 1977-1984 ;

- administrateur représentant le personnel, Fédération CGT des cheminots, 1979-1990 ;

- directeur du cabinet Fiterman, 1982-1984 ; président de RFF, 1997 2003 ;

- directeur de la coopération internationale de la SNCF, 1984-1990 ; - conseiller en communication des entreprises SEMA, SOFRES, BDDP, TBWA, 1976-2002;

- directeur régional de la SNCF, 1973-1976 ; directeur du Budget de la SNCF, 1983-1991;

- administrateur représentant de la FNAUT, 1983-1993;

- directeur des ventes à la direction commerciale Voyageurs de la SNCF, 1982-1989 ; directeur régional, 1990-1994 ;

- directeur général de la SNCF, 1988-1992;

- directeur général de la SNCF (2003), interrogé au titre de ses fonctions de directeur de cabinet du président (1989-1990), directeur de la Stratégie (1993-1996), directeur Grandes Lignes (1997-2003);

- président de la SNCF, 1988-1994;

- sénateur en charge du projet de loi sur la régionalisation des transports, 1994.

Par la position professionnelle, la série comporte neuf témoins occupant ou ayant occupé des fonctions à la SNCF (deux au conseil d'administration, six dans différentes directions, un à la tête d'un service spécifique) ; cinq personnalités extérieures (un parlementaire, un représentant de la tutelle, un représentant des usagers, deux représentants 
d'entreprises extérieures dont RFF). Le groupe constitué compte évidemment de nombreux absents qu'il est facile de remarquer en dressant, par exemple, la liste des présidents et des directeurs en mesure de témoigner sur cette période. Répétons que notre choix a tenté d'équilibrer de multiples perspectives thématiques en tentant de réduire au mieux la part d'aléatoire que comporte toujours le tracé d'un cercle de témoins. Ajoutons que cette répartition quantitative masque une distribution plus complexe car le choix des témoins reposait aussi sur les fonctions multiples qu'ils ont occupées.

\section{Le choix d'un questionnaire semi-directif}

Dans le cadre de cette étude, l'objet n'était pas de détailler chaque événement et d'en analyser les paramètres techniques, sociaux ou économiques. Il était entendu avec les témoins que cette série d'entretiens avait surtout pour ambition de réaliser un premier " tour de table». D’emblée était annoncé que chacun d'eux serait à nouveau interviewé de manière plus approfondie dans le cadre de la campagne définitive. Notre but était double. Nous voulions d'abord confronter avec la perception des "témoins-source» les dates majeures que nous considérions comme des points d'évolution ou de rupture et les thèmes à aborder pour faire une histoire des décisions stratégiques. L'entretien était ensuite le moyen de faire mentionner par les témoins les noms qui viendraient compléter le panel d'entretiens à réaliser durant la seconde phase du programme. La récurrence de certains noms cités a simplement confirmé l'utilité de leur présence au sein du corpus. En revanche, l'apport majeur a résidé dans l'ajout de témoins dont le repérage aurait été plus difficile car ils ne figuraient pas au premier plan des organigrammes consultés. Leur mention à ce stade de l'étude permet de réduire et de préciser le périmètre des entretiens.

La préparation des entretiens devait rester dans les limites d'une étude de faisabilité, que nous avons comprise à la fois comme un travail préalable permettant d'orienter les entretiens suivants et comme une épreuve des procédures et moyens mis en œuvre. Il aurait été disproportionné de consulter l'ensemble des archives que l'on mettra à profit durant la campagne d'archives orales pour réaliser des entretiens « test » dont la durée prévue contractuellement avec les témoins ne devait pas excéder deux heures. Nous avons donc formulé notre questionnaire à partir d'une bibliographie synthétique et de sources imprimées, rapports officiels ou commissions d'enquête ${ }^{10}$. Un travail essentiel a été effectué

10- Voir en fin d'article. 
en amont de l'enquête pour établir une chronologie synoptique de la période étudiée ${ }^{11}$.

Les archives orales, on le sait, peuvent être conduites selon des questionnaires systématiques, des récits libres, la restitution d'un parcours biographique, le fil d'une carrière. Nous avons choisi de réaliser des entretiens semi-directifs permettant de proposer des questions ouvertes à l'intérieur de grands thèmes (leur liste est fournie en annexe). La méthode garantit une approche à la fois comparative par le jeu des questions récurrentes et personnalisée en fonction de la carrière du témoin ou de la période chronologique évoquée. Directives par la recherche d'informations précises, les questions laissaient une latitude nécessaire au témoin pour élargir les perspectives proposées. La stimulation l'a ainsi souvent emporté sur un récit trop général. Les récits de carrière ont été minorés mais cette option n'a pas empêché pour autant l'insertion de quelques anecdotes menant l'entretien vers le récit de vie imbriqué à l'évocation des thèmes directeurs ${ }^{12}$. Tenir cette voie étroite était une contrainte pour des témoins dont la connaissance précise et vaste des thèmes abordés pouvait conduire à vouloir en dire beaucoup plus. Peu de dérives sont cependant à noter, notamment, comme nous l'avons dit, par l'acceptation contractuelle d'un second entretien détaillé lors de la seconde phase du programme. Aucune grille préalable n'a été fournie aux témoins, parce que nous souhaitions qu'ils restent attachés en priorité aux faits plutôt que de réfléchir à l'avance à leur interprétation ou à la façon de les étayer par un argumentaire.

\section{Une approche globalement satisfaisante}

Neuf témoins ont été entendus dans leur bureau, cinq à leur domicile. Parmi les neuf premiers, qui ont appartenu tous à la SNCF, six nous ont reçu au siège de leur activité actuelle, hors d'un « environnement SNCF »; deux autres au siège de la SNCF sur leur lieu de fonction, un au siège de RFF. La liberté de ton n'a guère été affectée par cette localisation des entretiens mais on peut bien sûr regretter que deux

11- Réalisée par madame Geneviève Chauveau, cette chronologie est établie à partir des rapports annuels d'activité de 1967 à 2002. Elle constitue dorénavant un instrument de travail très utile à tout chercheur. Elle reprend huit thèmes que nous avions définis comme des entrées possibles pour donner un cadre aux interviews : administration et statuts, politique et action commerciale, l'internationalisation de la SNCF, la recherche dans la coopération internationale, la SNCF et les régions, les transports en Ile-deFrance, investissements, projets et expérimentation, informatique et recherche. Geneviève Chauveau, « Rapports annuels d'activités. Chronologie, 1967-2002 », AHICF, 109 p. dactyl., janvier 2004.

12- F. Descamps, op. cit., p. 323. 
témoins, très sollicités sur leur lieu de travail, aient dû s'interrompre plusieurs fois. Prévus pour durer deux heures, les enregistrements n'ont en fait pas suivi exactement cette règle initiale. Ils ont varié entre une heure vingt pour le plus court et trois heures pour les plus longs (deux cas). La moyenne des quatorze entretiens s'est fixée à 115 minutes, 104 si l'on ne tient pas compte des deux entretiens de trois heures.

Peu de choses sont à signaler sur le plan matériel. Seul un entretien a été enregistré sur des cassettes analogiques de 90 minutes. Les autres l'ont été sur des cassettes DAT de 60 minutes, puis l'enregistrement transféré sur CD audio. L'apport d'un assistant technique a été un facteur de réussite indiscutable de la qualité technique des entretiens. Les témoins sollicités, habitués à intervenir en public, ne sont pas gênés par cette présence, perçue même comme un élément de prise en considération de la valeur de leur témoignage. Deux entretiens n’ont pas été enregistrés car, d'un commun accord avec les témoins, il était prévu de procéder à un balayage rapide des thèmes à explorer, en s'autorisant un propos plus libre.

Au total, l'étude de faisabilité a permis d'enregistrer 23 heures d'entretiens, dorénavant déposés à l'AHICF et consultables en fonction des prescriptions des témoins auxquels nous avons remis un contrat leur assurant le choix des modalités de diffusion. Celui-ci distingue les conditions de communication de l'enregistrement et celles relatives à l'éventuelle transcription de l'entretien. Il autorise le témoin à prescrire pendant un délai qu'il détermine ou à autoriser immédiatement la consultation individuelle de l'enregistrement, sa copie, son exploitation afin d'utiliser les informations dans le cadre d'une étude scientifique - sous la réserve évidente du respect de la vie privée des personnes nommées et des secrets protégés par la loi - et la diffusion sonore de tout ou partie de l'enregistrement.

Il en va bien sûr de l'intérêt des chercheurs de pouvoir bénéficier d'un accès immédiat au document sonore, mais la certitude d'un délai, même relativement court, peut permettre dans certains cas à un témoin de s'exprimer davantage sur des faits récents ou sur des évolutions en cours. Cette tendance générale s'est vérifiée dans notre cas : neuf témoins ont accepté la communication immédiate de leur propos, trois ont fixé à celle-ci un délai de trois à cinq ans après l'entretien, certains ont différencié la communication de l'entretien et celle de sa transcription, montrant leur préférence pour un discours entièrement contrôlé ${ }^{13}$.

13- Un seul a souhaité une transcription préalable à la signature du contrat de diffusion, procédure que nous souhaitons éviter par la suite tant pour des raisons de principe - il s'agit d'une collecte d'archives orales, les témoins doivent en être d'accord avant l'entretien - que de coût (risque encouru par l'AHICF de financer une transcription dont la communication est finalement refusée par le témoin). 


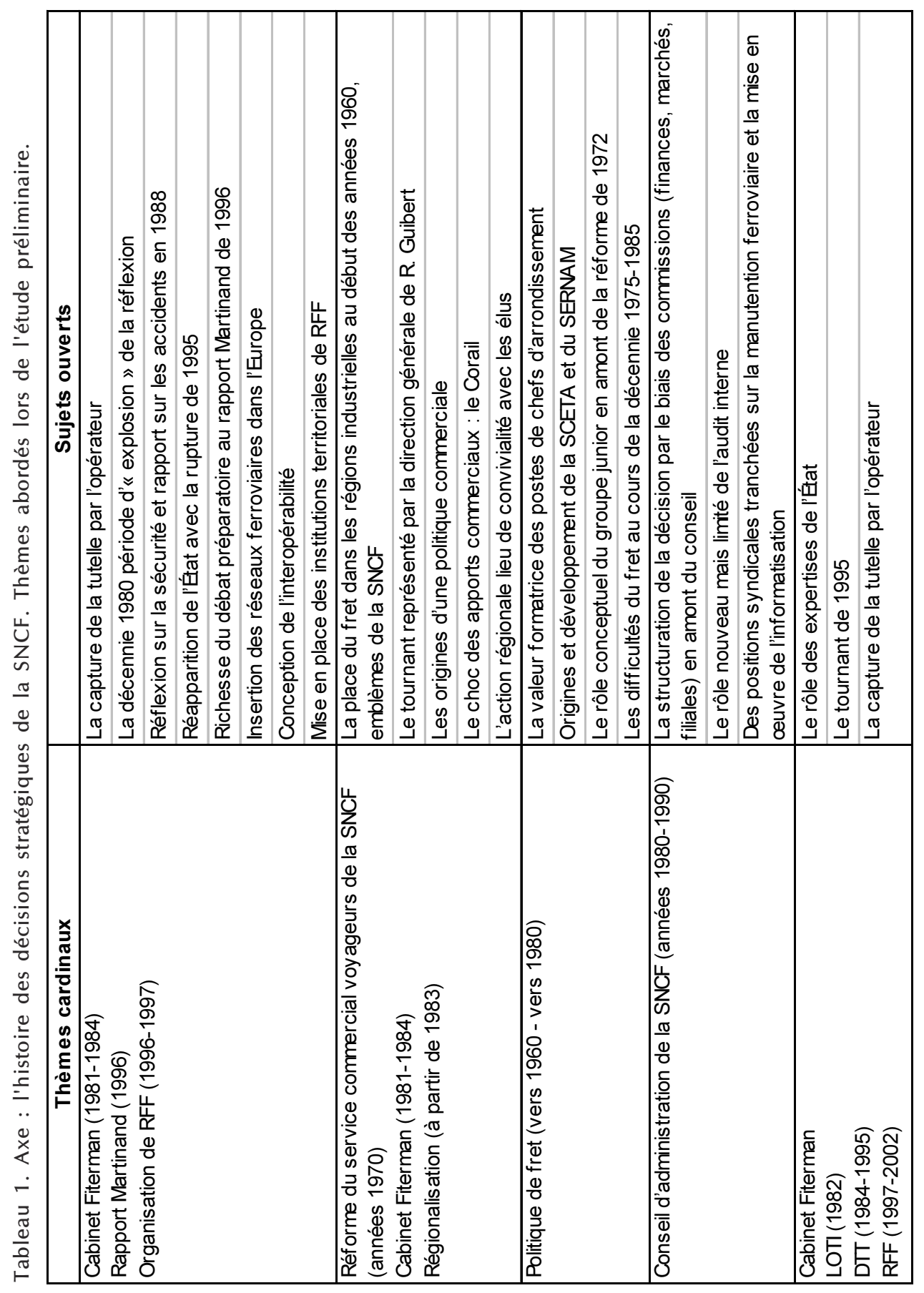




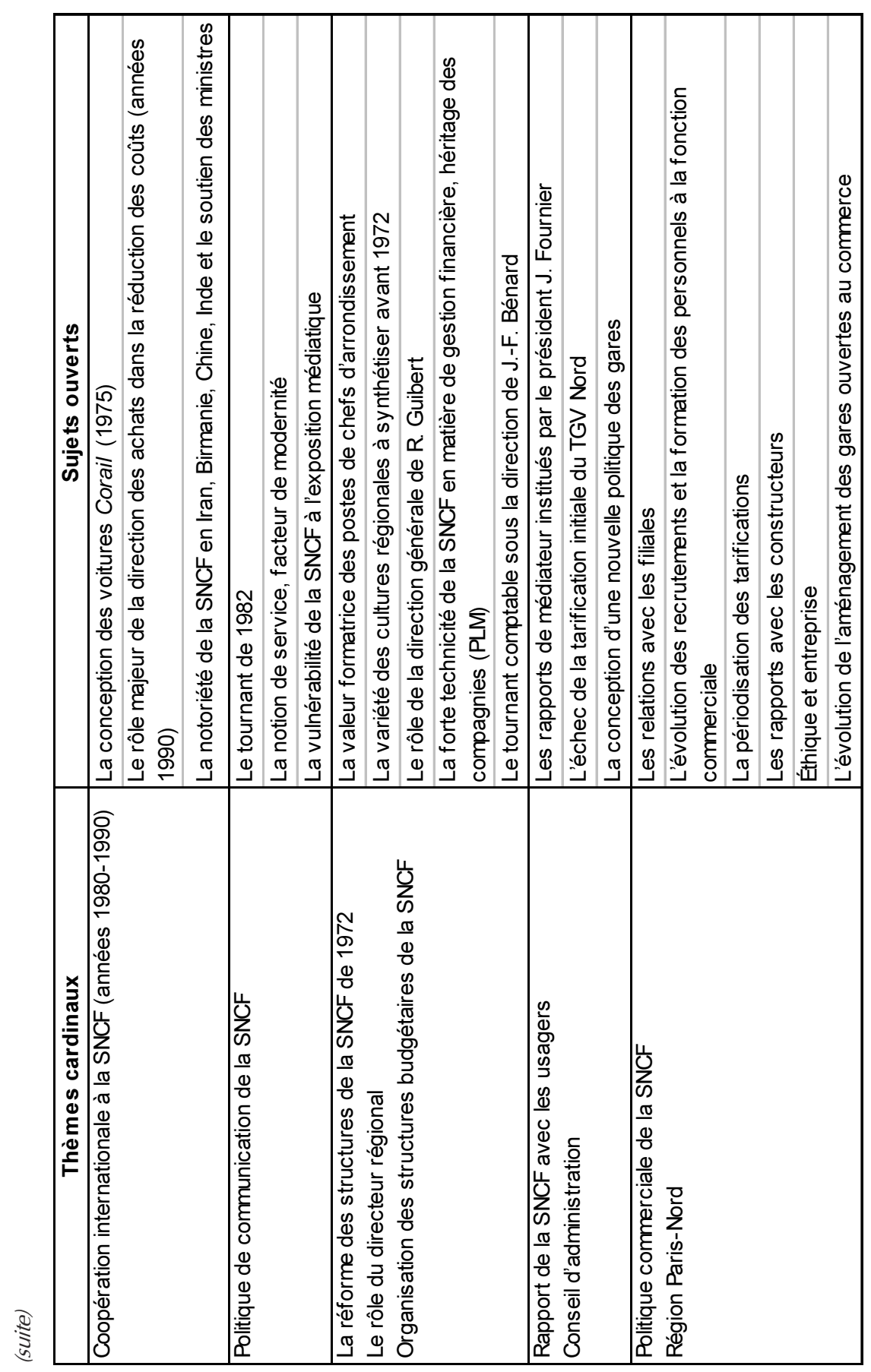




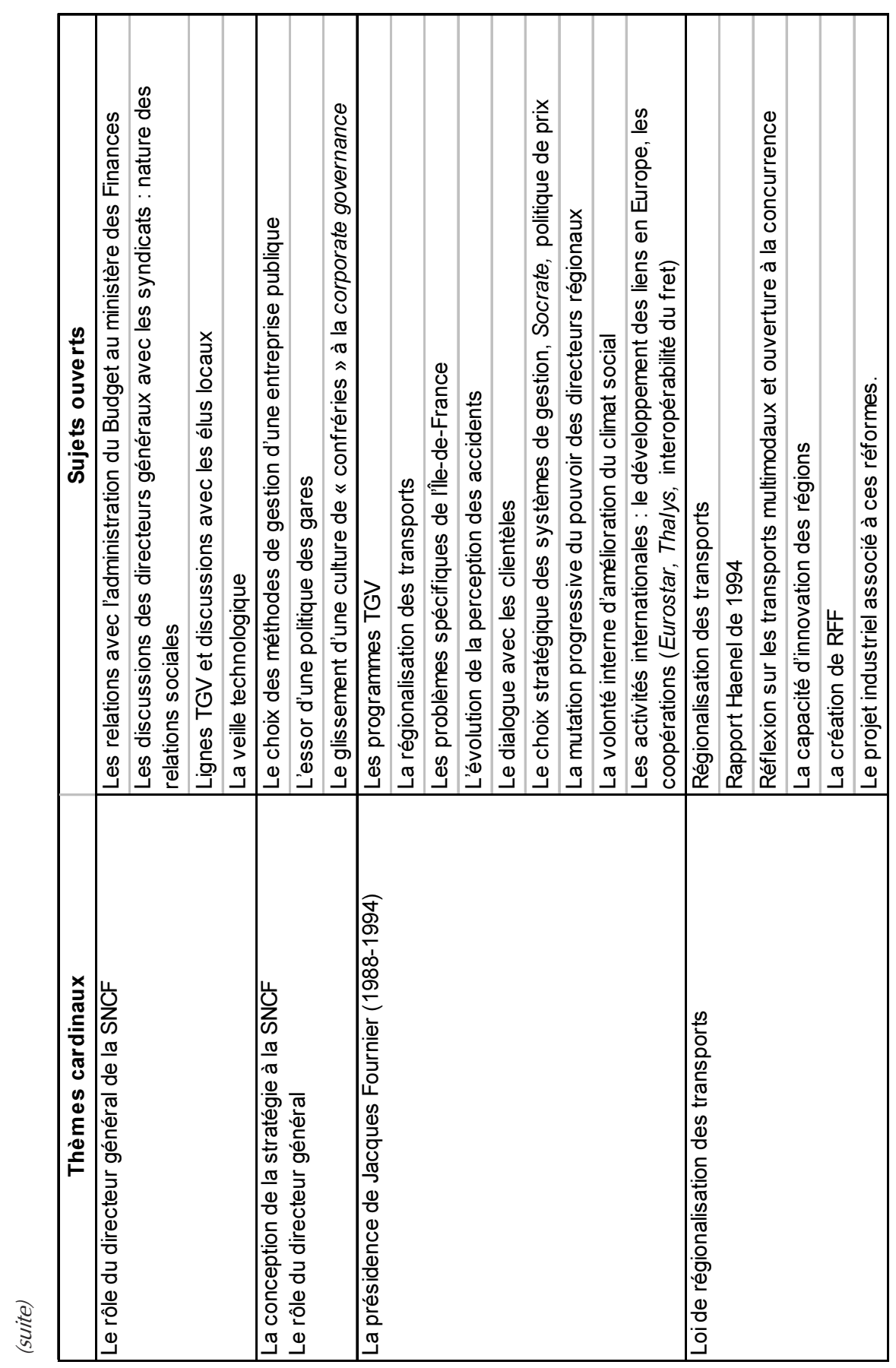


Les principaux thèmes qui ont fait l'objet de développements circonstanciés de la part de nos interlocuteurs ont permis de valider et de compléter les approches initiales. Le tableau ci-dessus permettra, sans révéler le contenu des entretiens, d'attester de la variété et de la richesse des pistes ouvertes.

\section{La délimitation d'un périmètre d'enquête}

Les thèmes envisagés permettent désormais d'affiner la problématique de la «décision stratégique » et de poser les cadres de la campagne de collecte d'archives orales à venir. Par décisions stratégiques nous entendrons l'ensemble des actions coordonnées dont on peut mesurer à long terme qu'elles ont orienté l'avenir de l'entreprise par des modifications structurelles. Trois questions se posent dès lors. La première est de préciser quels furent les acteurs principaux de la prise de décision et selon quelles modalités ils ont pu concerter leur action ou, au contraire, s'opposer sur un calendrier, des priorités techniques ou des arbitrages financiers. Cela amène à s'intéresser dans un premier temps plutôt à la source de la décision stratégique qu'aux différentes formes de son application. Une seconde interrogation consiste à analyser comment les structures organisationnelles de la SNCF ont été modifiées par l'effet de décisions qui se sont avérées stratégiques. La réflexion devra porter cette fois sur une lecture chronologique précise des transformations mais aussi des phénomènes d'acceptation ou de résistance de telles évolutions. Enfin, il faut déterminer comment les processus de changement au sein de la SNCF ont engendré l'évolution des cultures pour faire naitre des aspirations communes, creuset des orientations contemporaines de l'entreprise publique.

\section{Les acteurs des processus de décision stratégique}

Un premier point portait sur le rôle des ministères - et de la personnalité des ministres - pour dégager des inflexions politiques fortes. À ce titre, le ministère Fiterman (1981-1984) est bien perçu comme étant une période d'orientations nouvelles essentielles. Les contacts entre ses divers membres ont favorisé l'expression d'idées neuves aussi bien à propos de la place du transport routier que de la prise en compte du transport ferroviaire. Des membres du cabinet ont ainsi été portés à concevoir une transformation de la notion de service public. La réflexion du cabinet Fiterman s'est orientée également vers la recherche de gains de productivité, favorisant de nombreuses anticipations sur des évolutions constatées durant les années 1990. Bien que n’étant pas à proprement parler une "rupture », la LOTI (loi d'orientation des 
transports intérieurs, $\mathrm{n}^{\circ} 82-1153$, du 30 décembre 1982), par l'efficacité du travail engagé, peut être considérée comme un cadre encore solide des évolutions actuelles. Selon un des témoins, la notion de droit au transport ne semble pas avoir été poussée assez loin sur le plan juridique, restant surtout une notion politique, mais elle atteste d'un large débat d'idées. L'action du cabinet Pons-Idrac (mai 1995-juin 1997) est retenue comme un autre temps fort lorsqu'il prépare la révision du contrat de plan État-SNCF et qu'un projet de loi au large consensus parlementaire initial fait évoluer la situation de crise née en 1995 à la SNCF. On peut aussi l'attester par la confirmation essentielle des engagements lors du ministère Gayssot (juin 1997-mai 2002). De façon plus générale devra être mesuré le rapport - souvent, classique, de tension - entre le ministère en charge des Transports et le ministère des Finances dont plusieurs témoins estiment qu'il a repris sa prépondérance en 1983, imposant par ses arbitrages des choix politiques en à-coups, dommageables tant dans le domaine du fret que dans le schéma de construction des lignes nouvelles à grande vitesse. En cernant les acteurs des décisions stratégiques, il est apparu que la direction des Transports terrestres tenait finalement une place modeste dans le domaine ferroviaire par rapport à l'influence qu'elle exerce sur le transport routier. De même, les relations du secteur ferroviaire avec la DATAR ont fluctué, allant plutôt dans le sens d'une rétraction durable de cet organisme. La DTT a vu ses responsabilités diluées dans les années 1970 et est revenue au premier plan seulement en 1982. Quant à la DATAR, si elle joue un rôle influent pour promouvoir la décentralisation, elle ne semble pas avoir animé une politique continue qui aurait conduit à l'adoption et à la mise en œuvre de schémas directeurs d'aménagement.

Le postulat de départ considérant le renforcement du pouvoir du président de la SNCF aux dépens de son directeur général comme un facteur de la décision stratégique est bien confirmé par les entretiens. En fonction des périodes proposées aux témoins, ressortent: la présidence responsable et forte d'André Ségalat (1958-1975) ; celle, marquante, d'André Chadeau (1981-1985) qui a tiré le pouvoir décisionnel vers la présidence alors qu'il était jusque-là plutôt centré sur le directeur général ; celle de Jacques Fournier (1988-1994), probablement plus ouverte à la concertation sociale, avec les cheminots comme avec les usagers. D'autres sont plutôt considérées comme des présidences de transition, comme celles de Jean Bergougnoux (19941995) ou celle de Loïk Le Floc'h-Prigent (1 ${ }^{\text {er }}$ semestre 1996). A contrario, si les directeurs généraux des dernières années considèrent que le président exerce tous les pouvoirs, les directions de Roger Guibert 
(1966-1974) et de Jean Dupuy (1985-1987) sont toujours rappelées comme des temps forts du côté des directeurs généraux. Le renforcement des pouvoirs du président face au directeur général d'une part, l'évolution du rôle des directeurs régionaux, un temps chargés (1988-1994) d'établir un pouvoir local fort, vers le management d'équipe, d'autre part, ont complètement transformé le système de pouvoirs au cours de la quinzaine d'années 1981-1995. En filigrane de cette mutation apparaît un glissement de la culture technique de l'ingénieur vers celle, plus technocratique, du «commercial». En fait, il faut évoquer un nouveau dialogue entre technique et esprit commercial plutôt que d'insister sur l'effacement de certains pouvoirs. On pourrait partir par exemple de la responsabilité successive des différents services dans la conception des nouveaux matériels ferroviaires (Corail, 1976 ; TGV, à partir de 1981) pour valider cette hypothèse.

Outre le poids des acteurs évoqués, les processus de décision stratégique ont résulté d'interactions nouvelles avec les élus locaux qui prennent en compte l'aménagement (ou l'équipement) de leur territoire. Évidente dans le cas de la régionalisation, cette tendance est une conséquence des lois de décentralisation. Plusieurs témoins confirment que la difficulté, pour la SNCF, d'imposer des modifications dans la structure du réseau apparaît au début des années 1990. Elle est perçue comme une résurgence de l'influence que les élus locaux exercent pour garantir le maillage de leur territoire. Ce thème doit être étudié autant à travers la volonté de certains élus de garantir coûte que coûte une desserte ferroviaire, même déficitaire, que dans les combats menés par d'autres responsables politiques pour obtenir le raccordement aux lignes TGV (pourquoi Arras et non Amiens ?) ou pour relayer les pressions de l'opinion publique (tracés du TGV Sud-Est). On ne peut ainsi faire abstraction des conjonctures politiques ni des positions d'influence locales pour expliquer l'aboutissement ou l'échec de choix d'infrastructures ferroviaires à l'échelle du territoire.

\section{Les évolutions organisationnelles}

La mise en conformité de la SNCF avec les principes du management moderne pourrait être datée de la réforme des structures de 1972 que nous considérions comme un point de départ possible de notre période d'étude. Elle fut initiée par une réflexion découlant du rapport Nora (1967) sur les entreprises publiques. Dès 1968, le directeur général Roger Guibert ébaucha un programme appuyé sur la concertation avec les syndicats, à l'occasion de tables rondes, et mit en place une mission de coordination confiée à l'un des témoins interrogés. 
Au tournant de l'année 1969-1970 un groupe de réflexion «junior» ajouta ses propositions. La réforme, axée sur trois aspects (décentralisation des décisions, centralisation de l'exécution, coordination des éléments concourant à l'exploitation), déboucha sur des transformations essentielles dont l'évolution ultérieure a conditionné les décisions stratégiques. On retiendra en particulier :

- La création de vingt-cinq régions.

Celles-ci ne correspondent pas aux vingt et une circonscriptions d'action régionale mais remplacent les cinq anciennes régions $\mathrm{SNCF}$, issues des anciens réseaux, qui étaient placées sous l'autorité d'un directeur chargé de gérer le service dans le cadre des directives de la direction générale. Appliquée d'abord au réseau de l'Est et à celui du Sud-Est, la réforme est effective en 1973. Elle ouvre le débat sur les pouvoirs du directeur de région. Sous la tutelle des réseaux jusqu'en 1974, le pouvoir des directeurs régionaux est devenu effectif durant les années 1988-1994 avec la recherche d'une plus grande autonomie des directeurs face aux directions fonctionnelles de la direction générale (matériel et traction, équipement, transport...) ${ }^{14}$. Justifié par la complexité d'exploitation de certaines régions, cet ascendant est battu en brèche depuis lors, faisant évoluer le rôle du directeur de région plutôt vers la coordination managériale.

- La transformation des anciens services nationaux et de leurs arrondissements en directions techniques.

Les arrondissements apparaissent comme des foyers de formation essentiels. Ils structuraient géographiquement le découpage des services (exploitation, matériel et traction, voie et bâtiments). Chaque chef d'arrondissement portait, sur son territoire, les véritables responsabilités. Son autorité était relayée jusqu'aux établissements par des circonscriptions, des agences, des sections qui étaient des intermédiaires essentiels. Tel chef d'arrondissement du service voie et bâtiments se souvient d'avoir parcouru à pied l'intégralité de ses voies, acquérant ainsi une connaissance du terrain et des hommes capitale. C'était aussi un poste de représentation vis-à-vis de l'extérieur dont la notabilité insérait la SNCF dans la région. Bien que leurs témoignages soient difficiles à réunir aujourd'hui, les anciens chefs d'arrondissement pourraient apporter une connaissance détaillée de la SNCF au début de la décennie 1970.

14- Sur cet aspect, on s'appuiera sur l'étude conduite par Georges Ribeill, «Des patrons aux managers. Réalités et faux-semblants des pouvoirs des directeurs de région ", Paris, CERTES-LATTS, ENPC, juin 1990, 114 p. 
- La réforme des structures de commandement.

L'établissement d'une hiérarchie nouvelle a résulté de cette réforme (directeur général assisté par un directeur général adjoint, création de directions dont les pouvoirs se sont accrus par transfert de compétences relevant auparavant des services régionaux, établissements). Si les réformes récentes ont remis au premier plan les établissements, la réforme de 1972 peut être considérée comme ayant eu des effets structurants forts par le biais des directions régionales. On doit ainsi s’interroger sur l'échec du regroupement des régions souhaité par Jacques Fournier à la fin des années 1980 pour en réduire le nombre.

La mise en place du statut d'EPIC (établissement public à caractère industriel et commercial) en 1982, année charnière, puisque dernière année d'application de la convention du 31 août 1937 qui avait créé la SNCF société d'économie mixte, est un autre tournant. Elle engendre des conséquences importantes à long terme. Le statut prévoit de nouvelles modalités concernant le régime juridique et financier de la SNCF, qui conserve son nom, le contrôle économique et financier exercé sur elle par l'État, les élections et nominations des représentants au conseil d'administration, la création du Conseil national des transports et des comités régionaux et départementaux. Le choix d'un EPIC a suscité des résistances, certains craignant que l'État n'apporte pas de véritables garanties en matière de consistance du réseau. Pourtant, il a aussi permis des évolutions renforçant les relations avec l'État. Plusieurs groupes de travail (tutelle, SNCF, syndicats) ont étudié entre décembre 1981 et mars 1982 les éléments fondateurs de ce nouveau statut : définition du service public, autonomie de gestion par centre de responsabilité, décentralisation de la décision. Il conviendrait d'en recueillir systématiquement la mémoire.

Si les réformes institutionnelles créent des cadres qui contraignent l'action, la réorganisation de l'entreprise fait apparaître des changements d'organigramme nombreux durant cette période. La chronologie est à cet égard signifiante de besoins nouveaux, inséparables des préoccupations stratégiques et des logiques décisionnelles. Les entretiens ont ainsi confirmé l'enjeu que représentaient la création d'une direction de la Communication en avril 1982 ; celle, en 1987, d'un contrôle de gestion à la direction générale ; la courte existence d'un groupe de prospective entre 1983 et 1989 ; la création d'une direction de la Stratégie en 1991 ; l'adoption d'une direction de l'Action régionale en 1993, succédant au Service de l'action régionale apparu en 1983. L'étude de chacune de ces créations s'avère indispensable pour saisir les inflexions 
spécifiques de cette période. Par exemple, l'émergence d'une direction de la Recherche en 1991 rattachée au directeur général adjoint apparait comme une volonté de créer des synergies, non sans difficulté, entre la nécessité de maintenir des recherches par direction fonctionnelle pour assurer leur capacité d'innovation et la mise en œuvre d'une veille technologique généralisée, un dilemme rencontré en d'autres termes dans les années 1960 avec le Service de la recherche qui a coordonné le projet de TGV. D'autres attestent la prise en compte par l'entreprise d'aspirations comportementales et sociétales contemporaines lorsque sont instaurés successivement la direction des Audits (1990), l'inspection générale, l'Institut du management ou la fonction de contrôleur général chargé d'une mission d'éthique (définition et prescription de règles de comportement professionnel, 1998).

La loi du 13 février 1997 est porteuse d'une rupture marquante, avec la création de Réseau ferré de France (RFF). Son rôle était d'établir une programmation du financement et de la réalisation des investissements sur le réseau ferré, d'assurer l'entretien et l'exploitation des réseaux, d'identifier les besoins avec la SNCF et d'exercer ou de déléguer la maittrise d'ouvrage, enfin de confier par contrat à la SNCF, société de transport, la gestion du trafic, la surveillance et l'entretien des installations. Parmi les objectifs figurait notamment la volonté de modérer les investissements et d'éviter la « dérive financière antérieure » ${ }^{15}$. C'est assez dire combien cette date doit être considérée comme essentielle. La fondation de RFF doit être inscrite dans un déroulement d'événements : transposition dans le droit français de la directive européenne 91-440 (séparation des comptes des infrastructures et des opérateurs des réseaux), évolution du déficit qui s'est creusé entre 1989 et 1995 par la réalisation d'un schéma ambitieux de LGV et, selon l'un des témoins, l'adoption, lors d'une période euphorique, d'une grille salariale surévaluée qui mésestimait le retournement de conjoncture en cours. Confirmée par le ministère Gayssot qui avait pourtant déposé plus de 200 amendements contre cette création, l'introduction de RFF dans le paysage ferroviaire français est un seuil qui pourrait déterminer des changements radicaux. Après sept années d'existence, l'appréciation du fonctionnement de RFF reste un domaine de forte incertitude, liée aux difficultés de financement (faute d'un modèle économique d'organisation qui puisse être simplement adapté) et aux aspects institutionnels (la position de RFF n'étant justifiable qu'avec une vraie concurrence). Histoire « à chaud », celle-ci doit cependant être incluse

15- Claude Martinand, «Réseau ferré de France: missions, activités et perspectives d'avenir », RGCF, octobre 1998, p. 6. 
dans le programme afin de se donner les moyens plus tard d'en saisir la perception qu'en eurent les contemporains.

\section{L'adaptation de la culture d'entreprise à de nouvelles règles du jeu}

La naissance de la fonction commerciale à la SNCF est revenue au cours de plusieurs entretiens comme étant sans doute l'évolution stratégique la plus importante. Perçue comme l'une des évolutions capitales de l'entreprise, elle procède des initiatives qui ont été lancées en particulier par Jean Ravel, dont le nom est systématiquement cité dans les entretiens, au début de la décennie 1970. Selon un témoin proche de ces mutations, l'entreprise était jusque-là pilotée par la technique et le domaine commercial se réduisait à la gestion des tarifs. En amont, un ensemble de mesures significatives prises dans la mouvance du rapport Nora de 1967 rencontrèrent, à l'intérieur de la SNCF, la volonté de Roger Hutter (directeur général adjoint, 1966-1976) dont la pensée économique le poussait à rationaliser l'entreprise. Le tournant date des décisions de Roger Guibert de lancer en 1967 des études commerciales. Plusieurs mesures découlent de nouvelles approches : constitution d'une équipe pluridisciplinaire (apport des sciences humaines et de la sociologie) ; création d'une filiale publicitaire en 1975 ; division de la direction en deux parties (voyageurs et marchandises) en 1977 dont l'aboutissement conduit à distinguer direction des Grandes Lignes et direction du Fret. Le départ de Jean Ravel en 1981 crée un nouveau tournant, avec la prépondérance retrouvée des mesures quantitatives aux dépens d'une approche de marketing. Celle-ci avait introduit une conception de la politique commerciale radicalement nouvelle mais le changement mit vingt ans à se faire si l'on s'appuie sur l'embauche des diplômés de HEC (admise dans les années 1980) ou l'arrivée de directeurs régionaux à formation non technique (seulement avec la fin de la décennie 1990). Dans la durée, la fonction commerciale est indissociable des choix stratégiques qui ont été opérés dans deux domaines spécifiques.

La politique de tarification, conçue comme un ajustement à la demande, a multiplié les formules nouvelles : tarif bleu-blanc-rouge ; carte Vermeil, tarif Rail-Europe-junior (1971) ; Joker (1987) ; Carrissimo; simplification vers les tarifs réduits à $25 \%$ et à $50 \%$. Son histoire met en lumière l'alternative permanente entre politique de volume, difficile à mettre en œuvre lorsque dans la seconde moitié des années 1990 le manque de capacité matérielle l'entrave, résolue après 2001 par l'arrivée des rames duplex, et politique de marge, responsable de l'échec de la tarification d'abord adoptée sur le TGV Nord. Observées dans la longue 
durée, les décisions stratégiques ne se résument donc pas à des initiatives efficaces comme l'adoption avec la RATP de la Carte orange en région parisienne (juillet 1975) ou à des échecs médiatisés comme le lancement du système Socrate (1993).

Cette période est par ailleurs marquée par la constitution d'un nouvel environnement du voyage. Les messages de communication, appuyés sur les études de clientèles, sont renouvelés. Parfois utopiques au regard des difficultés pratiques de fonctionnement qu'entraînait par exemple la formule «Échappée belle », ils traduisent à la fin des années 1980 une volonté de transformation du service à l'image de la campagne «C’est possible! » centrée sur la qualité de l'offre. De même, les démarches de concertation organisées sous la présidence de Jacques Fournier (service de médiation, charte passée entre la SNCF et les associations de consommateurs de 1990, périodiquement renouvelée) renouent des liens avec la clientèle après la période de crise des années 1992-1995. La transformation de l'espace des gares apparait comme un champ de décisions stratégiques très novatrices. Née dès le milieu des années 1970 avec la séparation fret/voyageurs, matérialisée par des ruptures comme la substitution du compostage des billets au contrôle manuel en 1978, l'adaptation des gares engendre une perception différente du voyage. Plusieurs thèmes doivent être étudiés : le programme de rénovation qui commence en 1978 (Bordeaux, Douai, Dijon...) ; l'édification des gares TGV ; l'évolution de la conception de l'accueil du voyageur, avec par exemple la suppression critiquée des salles d'attente ou l'ancrage des espaces commerciaux dans l'espace ferroviaire à l'image de ce qui se faisait dans les aéroports et dont l'influence sur l'évolution de la relation avec les clientèles à la SNCF parait indéniable. La création d'une direction du Développement des gares en 1996 est donc autant un point de départ qu'un point d'arrivée de la concrétisation de la place reconnue aux gares comme lieu stratégique de la fonction commerciale.

Dans le temps même où la politique commerciale consacrait la croissance du trafic voyageurs ${ }^{16}$, la politique de fret subissait les contrecoups d'une évolution inverse. Alors qu'au milieu des années 1960 les arrondissements de Lille ou Metz étaient encore des postes considérés en raison du volume de fret lié aux activités industrielles, le recul est

16- Rappelons qu'entre 1970 et 1994, le trafic voyageurs total passe de 45 milliards de voyageurs/kilomètres à 65 , gagnés grâce au TGV, le point culminant du trafic Grandes Lignes classiques culminant en 1981 et décroissant après cette date. 
devenu régulier depuis le milieu de la décennie $1970^{17}$. Il faut bien sûr corréler cette tendance avec la concurrence accrue du poids lourd. Chronologiquement, elle correspond à la mise en œuvre sous Georges Pompidou d'une dynamique de construction autoroutière. Mais d'autres explications sont avancées par les témoins pour expliquer le déclin du fret SNCF. La mise en œuvre du SERNAM (qui aurait pu être, dans l'esprit de son concepteur, une société au même titre que d'autres sociétés filiales composant le groupe SNCF, à l'instar de la SCETA) est interprétée par l'un d'eux comme une décision à moindre coût. Elle permettait de mieux comprendre les métiers concurrents sans pour autant porter une vraie dynamique commerciale. Les choix opérés dans la clientèle, accordant une prime au rapport tonne/kilomètre, n'ont pas à terme favorisé le fret dont le recul a été aggravé par les grèves, les retards et une logistique insuffisante. Pourtant, tel autre témoin avance que le SERNAM, par la nouveauté de ses structures, peut être considéré comme un organisme porteur d'innovations. Tel autre suggère que l'absence d'une volonté suivie de l'État a handicapé une politique de trafic de marchandises ambitieuse. L'étude du fret reconnaît des événements auxquels on peut accrocher une chronologie : création du SERNAM, abandon de certaines fonctions (STEF, transports frigorifiques), projets Commutor (1989-vers 1996), ouverture du fret à la concurrence en 2003 sous l'impulsion de la commission européenne. Mais elle ne saurait être comprise sans une mise en perspective du mouvement de désindustrialisation de la France depuis le milieu des années 1970, ni sans prendre en compte l'évolution des modes de distribution ou l'aménagement des pôles commerciaux (cas du choix de Rungis associé à la desserte autoroutière).

L'évolution de la conception des matériels justifie une autre approche des politiques commerciales. Combinée à une histoire des choix techniques, elle ouvre sur la connexion entre culture des ingénieurs et culture commerciale. Lorsque les matériels des anciennes compagnies arrivèrent au terme de leur exploitation au début des années 1970, le choix des nouveaux matériels suscita des stratégies différentes. Plusieurs témoignages inclinent à prendre la période 1966-1972 comme un temps de grande mobilisation innovante dans le domaine de la traction (traction thermique, turbotrain, matériel « C03»-futur TGV -, développement

17- Le trafic de marchandises passe de 67 milliards de tonnes/kilomètres en 1970 à son sommet en 1974 avec 73 milliards de TK. Il décline ensuite avec des soubresauts entre 1975 et 1980 puis régresse fortement depuis cette date. En 1994, le trafic marchandises atteignait moins de 50 milliards de TK. En 2003, le transport ferroviaire des marchandises représentait $4 \%$ du transport total en France. 
du Service de la recherche, compétition avec le projet Bertin d'aérotrain, renonciation à la turbine à gaz qui laisse la place au TGV électrique). Dans le domaine du matériel, la conception des trains Corail, avec le designer Tallon, fut un tournant essentiel. Dotée de nombreuses innovations (climatisation en seconde classe, disposition sans compartiment), la voiture Corail-dont les témoignages de commerciaux et de techniciens tendent à parts égales à s'approprier la primeur introduisit une rupture dans le voyage. À cet égard, son adoption en 1975 doit être considérée comme une vraie décision stratégique. Qu’il s'agisse de relation avec le designer, d'innovation dans l'offre à la clientèle ou d'image de communication associée, un parallèle peut être avancé avec la conception, après 1990, des nouveaux TER. Dire que l'autre décision stratégique essentielle fut l'adoption des grandes vitesses est un truisme qui ne suppose pas de développement. Il va de soi qu'à tous égards - politique d'aménagement territorial, réduction de l'espacetemps, bouleversements de la hiérarchie urbaine - la mise en service du réseau de TGV fut non seulement porteuse de choix essentiels mais aussi le support d'une culture d'entreprise. Les relations avec les constructeurs (politique de développement du TGV ; mise en service des nouveaux TER) ont connu des évolutions qui restent à préciser. Ceci suppose d'intégrer des témoins de ces entreprises dans le programme. En revanche, la bonne connaissance de l'histoire du TGV grâce aux travaux que nous avons cités dispense de revoir ce qui est déjà su.

D'autres changements techniques apparaissent dans la gestion des réseaux. C'est le cas par exemple lors de la mise à l'étude en 1986 du système ASTREE (automatisation du suivi de localisation des trains par radars embarqués : prévention des accidents, amélioration de la productivité du réseau par accroissement sécurisé du trafic grâce au couple informatique - télécommunications). Il en va de même avec le SACEM (système d'aide à la conduite, l'exploitation, la maintenance) et le programme d'automatisation de l'exploitation (pilotage, contrôle de vitesse, signalisation en cabine) développé avec la RATP en 1988.

Le fruit de ces évolutions techniques et commerciales doit être aussi considéré à l'aune des changements introduits dans la desserte ferroviaire du territoire. Les sources de la régionalisation peuvent être recherchées dans les idées décentralisatrices d'Olivier Guichard qui a préparé, depuis la DATAR, la mise en place des EPR (établissements publics régionaux, loi du 5 juillet 1972). Dans ce mouvement accordant plus de responsabilités aux régions, on s'attendait à voir les élus locaux prendre des décisions sur l'aménagement des infrastructures régionales. Or, contrairement à l'hypothèse émise alors de la fermeture des lignes 
ferroviaires déficitaires, les régions ont souhaité renforcer le contrôle de leur développement. Dès 1977, des régions furent désignées pour une expérience de décentralisation (Lorraine, Pays-de-la-Loire, NordPas-de-Calais) pour qu'elles s'impliquent dans le financement et la définition des dessertes devant le succès des omnibus. En 1978, la région Nord-Pas-de-Calais entamait une évolution, signant la première convention avec la SNCF. Le rôle d'élus politiques tels le sénateur communiste Viron doit être rappelé avant même que les lois de 1982 n'incitent, plus généralement, à leurs interventions. Du côté de la SNCF, le tournant de la LOTI, malgré l'hostilité des Finances, incitait à prendre des initiatives, dont la création du Service d'action régionale en 1983 pourrait marquer une date-clé. La régionalisation correspondait à une attente des élus en quête de solutions face à l'essor de la mobilité de proximité au début des années 1980. L'élaboration d'une politique régionale sous l'impulsion de Jacques Chauvineau a rencontré cette demande, s'appuyant sur des liens d'équipe anciens. En 1986, dix-sept régions avaient signé des conventions avec la SNCF pour gérer et organiser des dessertes ferroviaires régionales dans le cadre des contrats de plan. Le rapport de l'entreprise aux régions changeait avec la prise en compte d'une concertation locale. L'une des conséquences les plus marquées a été la généralisation des TER ou Transports Express Régionaux. En 1987, à la création des TER, onze régions adhèrent rapidement à trois engagements : modernisation des réseaux, produits performants, politique d'image forte. Le second tournant est lié au rapport Haenel (1994) qui est venu accentuer cette politique d'initiative et a débouché sur la LOAT (loi d'orientation pour l'aménagement et le développement du territoire du 4 février 1995), renforcée par le ministère Gayssot (2000). La réussite de cet engagement envers les régions se traduit aujourd'hui dans les chiffres. Entre 1997 et 2002, la hausse du trafic a atteint $21 \%$. Les régions ont investi dans des matériels neufs, alors qu'une enquête montrait en 1996 que le matériel de plus de vingt ans représentait $21 \%$ du parc en Alsace, $18 \%$ en Provence-Alpes-Côte d'Azur, $12 \%$ dans le Nord-Pas-de-Calais. A posteriori, l'action engagée dès 1978, structurée depuis 1983, apparaît bien comme un choix stratégique, au point d'être considérée aujourd'hui comme une sortie « par le haut» de l'alternative maintien ou fermeture des petites lignes.

L'internationalisation des activités, une autre dimension de l'entreprise, a été également porteuse de décisions stratégiques. Elle suit deux voies principales. La première est la participation aux grands projets transnationaux : mise en œuvre du tunnel sous la Manche par convention en 1973 mais non ratifiée, débloquée par la signature du 12 février 1986 ; 
organisation d'un réseau européen à grande vitesse proposée en 1982 lors du sommet franco-allemand qui aboutit en 1985 au Paris-Cologne ; mise en œuvre de Eurostar et de Thalys. L'exportation du savoir-faire d'ingénierie est un autre canal de cette internationalisation. Réalisée par le bais de Sofrerail, Société française d'études et réalisations ferroviaires, devenue Systra en 1992 par regroupement avec la filiale Sofretu de la RATP, appuyée sur la création en 1980 d'un département de la Coopération internationale devenu en 1997 une direction du Développement international, cette action parait récurrente. On relèvera malgré tout le temps fort de la décennie 1980, vouée à la promotion du TGV vers les pays d'Asie, avec le soutien des ministres des Transports, de la DREE (direction des Relations économiques extérieures aujourd'hui incluse dans la direction générale du Trésor et de la Politique économique) du ministère des Finances, le rôle ponctuel de certaines personnalités (ambassadeurs) et la concertation établie avec les industries ferroviaires. Pour autant, certains notent que cette internationalisation est trop limitée par défaut d'interopérabilité et souvent en raison des cultures ferroviaires propres à chaque entreprise.

\section{Conclusion : de la faisabilité aux préconisations}

Avoir consacré plus d'une vingtaine d'heures d'enregistrement à valider des postulats scientifiques pourra apparaitre comme une grande perte de temps et un coût supplémentaire dans la mise en œuvre d'une campagne d'archives orales ${ }^{18}$. On pourra aussi déplorer une sollicitation abusive des témoins au regard des hautes fonctions qu'ils ont exercées ou exercent encore pour certains d'entre eux. Il nous semble pourtant que trois apports liés à cette étude de faisabilité peuvent être mis en exergue, qui en font dans une certaine mesure une étude préliminaire, une première étape de notre programme.

Le premier enseignement est de disposer d'une chronologie pondérée assez fiable qui mêle histoire des structures, histoire technique et histoire économique. Peut-être celle-ci sera t-elle révisée au fil des entretiens.

18- Dans l'ouvrage de référence qu'elle a consacré aux archives orales, et plus particulièrement dans la partie dévolue à l'ingénierie des campagnes d'archives orales, madame Descamps ne fait pas état d'expériences similaires. Pour notre part il s'agissait d'une expérience nouvelle puisque nous ne l'avions pas employée dans nos deux pratiques précédentes : 35 interviews ciblés et courts, abordant l'histoire générale de l'entreprise ou d'un de ses secteurs, pour rédiger l'histoire de Gaz de France avec Alain Beltran (Le Noir et le Bleu, Quarante années d'bistoire du Gaz de France, Paris, Belfond, 1992); organisation et participation à 36 entretiens d'une campagne d'archives orales réalisée pour l'Association Georges Pompidou de 1993 à 1996 (avec Noëlline Castagnez de 1994 à 1996). 
Elle offre cependant des thèmes auxquels accrocher des questionnaires qui donneront une homogénéité supplémentaire aux archives orales. Elle contrebalance la composition hétérogène du corpus.

Par ailleurs, le recensement des noms marquants - ou apparaissant comme tels dans la mémoire des témoins - couplée à notre propre travail de composition de la liste permet d'établir un panel représentatif approchant la centaine de noms. Celui-ci mêlera donc cadres de l'entreprise aux niveaux faîtiers de la hiérarchie managériale, responsables politiques ou membres des cabinets ministériels de tutelle - Transports, Équipement, Finances - membres de collèges d'experts (consultés par exemple dans les procédures décisionnelles du TGV) et personnalités extérieures à la SNCF. Les hésitations sur le choix d'une campagne qui prendrait en compte soit tout un niveau hiérarchique sans distinction, soit établirait une progression par grandes masses homogènes (les ministres des Transports, les présidents de la SNCF, et ainsi de suite) se sont soldées par une approche thématique transversale plus fructueuse. Répartie sur trois années, la campagne pourrait s'organiser en trois volets. L'an un sera consacré aux cadres structurels (réforme de 1972, statut de 1982, relations avec la tutelle, organisation des directions, création de RFF). L'an deux portera sur les choix stratégiques (politique commerciale, politique de fret, choix technologiques). L'an trois sur les bouleversements vécus à l'échelle des territoires (région Île-de-France, régionalisation, internationalisation). Les rôles diversifiés des acteurs et la pluralité d'approches des témoins pressentis permettra d'aller vers une histoire sociale de la décision qui reste en fin de compte la plus intéressante à conduire dans ce cadre.

Enfin, l'étude de faisabilité démontre qu'en engageant une collecte de témoignages sur ces années, l'AHICF s'ouvre une perspective panoramique d'histoire contemporaine. Sur le plan scientifique et problématique, la récurrence de l'évocation de ce thème dans les entretiens de la première phase invite à prendre en compte de manière systématique le lien entre décision stratégique à la SNCF et aménagement du territoire. C'est évident concernant le TGV, cela ne l'est pas moins dorénavant avec la régionalisation et la mise en œuvre du réseau TER. Mais on peut développer la même approche par exemple avec l'évolution du fret dans les années 1970, la mise en œuvre d'une politique commerciale adaptée à des demandes nouvelles (trains Corail), l'organisation des services de banlieue. On voit dès lors que la problématique pourrait glisser vers une analyse des mutations de la France contemporaine depuis les années 1970 à travers l'histoire des grandes décisions stratégiques à la SNCF (relations avec l'industrie, évolution des 
politiques publiques d'aménagement des transports, inflexion des missions de service public, prise en compte des mobilités nouvelles). Dans la période plus récente, l'évolution des réseaux met également en exergue la construction d'un maillage européen nouveau depuis le milieu des années 1980, pensé dès la décennie 1970 (étant entendu que l'Europe des chemins de fer existe par des pratiques techniques et des concertations professionnelles bien antérieures). Le programme doit donc s'inscrire aussi dans la quête historiographique de la construction des réseaux européens.

Au total, on le voit, les quatorze entretiens de cette étude de faisabilité ont dégagé l'horizon du chercheur et posé des points de repère utiles. La qualité du matériau analysé et archivé en fait, déjà, la première étape de la campagne. Le mérite en revient aux témoins qui ont accepté de se plier à cet exercice difficile : donner des informations avec spontanéité et réflexion, corriger les erreurs d'appréciation, enrichir les analyses, le tout de façon synthétique. Ils l'ont tous fait avec disponibilité et qualité d'écoute. Qu'ils en soient remerciés.

\section{Bibliographie synthétique}

\section{Ouvrages}

Renaud Abord de Chatillon, La Politique des transports en France : entrer dans le XXI siècle, Paris, Eska, 1994 ;

Jean Bergougnoux, Prenons notre destin en main, SNCF, 1994 ;

G. Bessay, SNCF, l'avenir se décide anjourd'bui : le cadre de la politique des transports de demain, IFRET, 1994 ;

Marcel Boiteux, Transports : pour un meilleur choix des investissements, Paris, La Documentation française, 1994 ;

Cabinet DEGEST, L'Expérimentation de la régionalisation des transports ferroviaires de voyageurs, Paris, CCE SNCF, 1997;

Marc Caremantrant, «Le Rail français en 1983, année de la nouvelle SNCF et des 2 heures par le TGV entre Lyon et Paris », Biblio-Rail, 1984;

Gilbert Carrère, Le Transport en France, PUF, 1997 ;

G. Dumartin, 1981 à 1991 :10 ans de transport, Ministère de l'Équipement, du Logement et des Transports, OEST, 1993 ;

Clive Lamming, Cinquante ans de traction à la SNCF. Enjeux politiques, économiques et réponses techniques, Paris, CNRS Éditions, 1997 ;

Georges Ribeill, Cent cinquante ans de chemin de fer français : vues synthétiques sur le système technique et le milieu professionnel ferroviaires à l'époque de la longue durée, Paris, Ministère des Transports, 1982 ; 
Jean-François Troin, Rail et aménagement du territoire : des héritages aux nouveaux défis, Aix-en-Provence, Edisud, 1995 ;

C. Soulié, Le Grand Livre du TGV, Paris, Éd. de La Vie du rail, 2002 ;

La Vie du rail, 1995-2010 : les transports en Ile-de-France, Paris, Éd. de La Vie du rail, 1995.

Articles

Jean Huet, "Histoire de la politique commerciale marchandises des chemins de fer français des origines à nos jours ", in Maurice Wolkowitsch (sous la dir. de), « Politiques commerciales des chemins de fer », RHCF, $\mathrm{n}^{\circ} 9$ (print. 1993), p. 21-36.

«Les très grandes vitesses ferroviaires en France », RHCF, n¹2-13 (1995).

Michèle Merger et Michel Walrave (sous la dir. de), «Les Chemins de fer en temps de concurrence. Choix du XIX ${ }^{\mathrm{e}}$ siècle et débats actuels ", RHCF, n¹6-17 (1997).

Raymond Mourareau, «Dix ans d'interopérabilité, 1989-1999» (intervention à la table ronde réuni le jeudi 4 novembre 1999: "L'interopérabilité aujourd'bui et ses perspectives; vers l'Europe des chemins de fer? "), RHCF, n²6 (print. 2002), p. 199-206.

Enfin, plusieurs numéros de la Revue générale des chemins de fer (RGCF) ont été utilisés sur des thèmes précis :

Jean-François Picard ; Alain Beltran, « D'où viens-tu TGV ? Une histoire du TGV », RGCF, août-sept. 1994, p. 3-80 (Rapport de recherche, convention IHTP-AHICF, 1991-1992).

Pierre-Louis Rochet, "Systra-Sofretu-Sofrerail : 40 ans consacrés à l'optimisation des transports, besoin fondamental des économies modernes », RGCF, juin 1997, p. 25-27.

Jacques Chauvineau, «Les 200 jours de la régionalisation », RGCF, nov. 1997, p. 29-33.

Guillaume Pépy, "Le train plus simple et moins cher. Deux axes pour la reconquête commerciale voyageurs de la SNCF (1996-1998) ", RGCF, janv. 1999, p. 5-13.

Armand Toubol, "Le transport international de fret », RGCF, mars 1999, p. 5-10.

Jean-Louis Rohou, «Une voie française pour le renouveau du chemin de fer. La création de Réseau ferré de France », RGCF, juil.-août 1998, p. 31-38.

Jean-Michel Jomard, "RFF : cinq années d'une existence associée au renouveau du rail », RGCF, avril 2002, p. 39-42. 


\section{Rapports}

Paul Chollet, Le Transport des marchandises en Europe: sortir de l'impasse, Paris, Assemblée Nationale, 1994 ;

Henri Cuq et Dominique Bussereau, Une nouvelle donne pour la SNCF. Rapport de la commission d'enquête sur la situation de la SNCF, tome 1, Rapport; tome 2, Auditions, Paris, Assemblée Nationale, 1994 [5 témoins de notre liste ont donné une déposition] ;

Hubert Haenel, Régions, SNCF : vers un renouveau du service public : propositions, Ministère de l'Équipement, du Transport et du Tourisme, 1994 ;

Claude Martinand, Débat national sur l'avenir du transport ferroviaire, Rapport introductif du groupe de travail présidé par Claude Martinand, Paris, Ministère de l'Équipement, du Logement, des Transports et du Tourisme, 29 février 1996. 


\section{Annexe \\ Liste des thèmes choisis pour étudier I'histoire des décisions stratégiques à la SNCF depuis les années 1970}

\section{Les processus des décisions stratégiques}

\section{La réception de la réflexion des ingénieurs}

- Quelle est la chronologie de leur influence ?

- S'agit-il d'une influence en déclin face à la culture technocratique ?

La position des administrations centrales par rapport au secteur ferroviaire

- Les relations de la SNCF avec la direction des Transports terrestres - Les effets de la suppression, en 1982, de la direction générale des Transports intérieurs

- Partagent-elles une culture commune d'ingénieurs polytechniciens du corps des Ponts et Chaussées?

- Les relations de la SNCF avec la DATAR

- Les positions du Conseil d'État évoluent-elles, quand on considère ses réactions hostiles au choix des lignes TGV ?

\section{Les arbitrages des ministères}

- L'évolution de la position du ministère en charge de l'Équipement, des Transports et de l'Aménagement du territoire vis-à-vis du train et de la SNCF

- Les positions du ministère des Finances.

\section{L'aménagement du territoire}

- Le renforcement des dessertes intervilles en 1972 équivaut-il à une promotion des villes moyennes?

- Le choix pour un réseau TGV et ses modalités est-il l'aboutissement d'une culture ferroviaire française ? (électrification des lignes, responsabilité de l'État centralisateur en matière d'aménagement du territoire, rentabilité financière croisée avec avance technologique)

- L'arbitrage politique rendu en matière de transport de marchandises a-t-il été défavorable à la SNCF ? Si oui, quand?

- La LOTI (loi d'orientation des transports intérieurs) a-t-elle constitué une « rupture » en 1982, en instituant la notion de « droit au transport »? - Ce choix politique était-il partagé par les élus (députés des différentes tendances, sénateurs)? 


\section{Les évolutions des structures internes de la SNCF}

\section{La réforme de 1972}

- S'agit-il d'une mise en conformité de la SNCF avec principes du management moderne?

- Le programme de réforme ébauché dès 1970 a-t-il permis dans les faits comme il le prévoyait:

- une décentralisation des décisions?

- la centralisation de l'exécution?

- la coordination des éléments concourant à l'exploitation?

- La création de 25 régions

- Comment ont évolué les pouvoirs du directeur de région?

- Peut-on déceler une importance relative des régions dans les stratégies de carrière des directeurs régionaux et de divisions régionales?

- La réforme des structures de commandement.

\section{L'adoption du statut d'EPIC (1982)}

- La transformation du statut fait-elle de 1982 une année charnière?

- S'agit-il d'une nouvelle définition du service public?

- Quels sont les effets des élections et des nominations des représentants au conseil d'administration prévus par le nouveau statut?

- La création du Conseil national et des comités régionaux et départementaux des transports

- Bilan de la prévision de connexion des réseaux européens alors envisagée.

\section{La régionalisation des transports ferroviaires}

- Les conceptions commerciales des années 1970 et la fermeture des lignes déficitaires confrontées à la demande de mobilité locale (succès des omnibus)

- Les premières conventions avec le Nord-Pas-de-Calais puis dans le Midi sont-elles à l'origine des TER ?

- La LOTI et la collaboration avec les régions

- De 1984 au rapport Haenel (1994), émergence du pouvoir régional avec les TER ; création du Service d'action régionale de la SNCF

- Les obstacles à surmonter (hostilité des Finances, scepticisme de la SNCF, organisation pratique difficile)

- Les acteurs qui appuient le mouvement (élus régionaux, directeurs régionaux de la SNCF, ses présidents)

- Le rôle des «trains des régions» 
- 1987 est-elle une année tournant?

- La conception des TER

- Des années perçues concurremment à l'intérieur de l'entreprise comme celles du «tout TGV », comme celles où la direction Grandes Lignes décide de supprimer les petites lignes en contradiction avec les objectifs TER, et celles de l'essor de la régionalisation : enjeux stratégiques comparés des trois tendances

- Le rapport Haenel (1994)

- La loi d'orientation sur l'aménagement et le développement du territoire (1995)

- La réforme du 13 février 1997 (transfert à 6 régions de l'autorité organisatrice des services ferroviaires régionaux avec les ressources budgétaires correspondantes)

- La loi Gayssot du 14 décembre 2000 : généralisation de la régionalisation, effective depuis 2002.

\section{La naissance de la fonction commerciale}

- Quelle est la place du directeur général adjoint « commercial» dans la hiérarchie de la SNCF ?

- La division de la direction commerciale en deux (voyageurs/marchandises) puis la distinction entre direction des grandes lignes et direction du fret font-elles de 1977 une année tournant?

- Histoire des structures de concertation avec les usagers du chemin de fer.

\section{RFF en 1997}

- Quelles procédures de concertation avec la SNCF lors de l'élaboration de la loi et des décrets?

- La structuration de l'organisation de RFF

- Le rapport Martinand

- RFF réussit-il ce que ne pouvait faire la SNCF, et comment?

- Les critères de détermination des péages

- La corrélation avec la politique européenne d'accès des tiers aux réseaux

- Quelles nouvelles logiques pour transporter le fret?

\section{L'internationalisation des activités ferroviaires}

- La participation de la SNCF à la mise en œuvre du tunnel sous la Manche

- La proposition française au sommet franco-allemand de 1982 de mise en œuvre d'un réseau européen à grande vitesse qui aboutit en 1985 au Paris-Cologne 
- Eurostar et Thalys sont-ils des facteurs d'intégration européenne?

- L'évolution de la direction du développement international de la SNCF

- Le rôle joué par Sofrerail : promotion des techniques ferroviaires ou réseaux d'ingénieurs?

La naissance de services et grandes directions de la SNCF

- La réorganisation de 1972

- 1977 : la direction de l'Audit et de l'inspection générale

- La direction de la Communication : avril 1982

- 1987 : le contrôle de gestion de la direction générale

- 1983-1989 : la courte existence d'un groupe de prospective

- Du Service d'action régionale (1983) à la direction de l'Action régionale (1993)

- Le rôle de la direction de la Stratégie créée en 1991

- Le tournant de 1995 : recomposition du premier niveau managérial.

\section{De nouveaux engagements basés sur des ruptures ou des mutations?}

\section{Évolution technique des modes de traction et des matériels}

- Les grandes vitesses

- Les matériels voyageurs (conception des trains Corail mis en service en 1975 ; relations avec les constructeurs : politique de développement du TGV ; mise en service des nouveaux TER)

- 1986, tournant technologique ? Mise à l'étude du système ASTREE (automatisation du suivi de localisation des trains par radars embarqués) - Mise en œuvre du SACEM (système d'aide à la conduite, l'exploitation, la maintenance) et programme d'automatisation de l'exploitation (pilotage, contrôle de vitesse, signalisation en cabine) développé avec la RATP (1988).

\section{Politique commerciale}

- La conception d'une stratégie commerciale (périodisation)

- 1975 : la création d'une filiale publicitaire

- Le contrat de plan 1985-1989 (modernisation de l'offre traditionnelle; adaptation " pointue » des services et produits à la diversité des clients)

- La signature en 1990 d'un accord avec 19 associations nationales visant une charte de qualité et une amélioration du service

- La gestion du système SOCRATE (1990-1992, mise en service 1993) 
- La politique de communication (périodisation)

- L'évolution des formes de tarification

- La transformation de la conception des gares

- La gestion de la banlieue parisienne.

\section{Politique de fret}

- Création du SERNAM (1970)

- Valorisation ou anonymat du record mondial d'un train de fret en $1988(225 \mathrm{~km} / \mathrm{h})$ ?

- Le projet Commutor (1989) et la gestion robotisée des chargementsdéchargements couplés avec une gare routière

- L'ouverture du fret à la concurrence en mars 2003.

Spécificités de la culture $d^{\prime}$ ingénieur et culture technocratique

- Le rôle des bureaux d'étude

- La conception des matériels

- La perception du passage d'une culture d'ingénieur à une culture technocratique : de quand la dater?

\section{Les crises sont-elles vecteurs de ruptures?}

- Les grands conflits sociaux : 1986-1987; 1995

- Les accidents. 